\title{
Traction Control of Electric Vehicles Using Sliding-Mode Controller with Tractive Force Observer
}

\author{
Suwat Kuntanapreeda \\ Department of Mechanical and Aerospace Engineering, Faculty of Engineering, King Mongkut's University of \\ Technology North Bangkok, Bangkok 10800, Thailand \\ Correspondence should be addressed to Suwat Kuntanapreeda; suwat@kmutnb.ac.th
}

Received 24 June 2014; Revised 26 November 2014; Accepted 1 December 2014; Published 21 December 2014

Academic Editor: Nicolas Hautière

Copyright (C) 2014 Suwat Kuntanapreeda. This is an open access article distributed under the Creative Commons Attribution License, which permits unrestricted use, distribution, and reproduction in any medium, provided the original work is properly cited.

Traction control is an important element in modern vehicles to enhance drive efficiency, safety, and stability. Traction is produced by friction between tire and road, which is a nonlinear function of wheel slip. In this paper, a sliding-mode control approach is used to design a robust traction controller. The control objective is to operate vehicles such that a desired wheel slip ratio is achieved. A nonlinearity observer is employed to estimate tire tractive forces, which are used in the control law. Simulation and experimental results have illustrated the success of the proposed observer-based controller.

\section{Introduction}

Electric vehicles (EVs) have become very attractive in replacing conventional internal combustion engine vehicles because of environmental and energy issues. They have received a great attention from the research community. Control methodologies have been actively developed and applied to EVs to improve the EVs performances [1-8].

Traction control plays an important role in vehicle motion control because it can directly enhance drive efficiency, safety, and stability $[9,10]$. Traction is the vehicular propulsive force produced by friction between tire and road. The characteristics of the friction are nonlinear and uncertain, which make traction control difficult. The friction depends on many factors such as tire type, road surface, road condition, and wheel slip. Accordingly, an objective of the traction control is to operate vehicles such that a desired wheel slip ratio is obtained. The slip ratio yielding the maximum friction coefficient is usually desired because it yields the maximum torque from the propulsion system to drive the vehicle forward.

Traction control of electric vehicles has drawn extensive attention since electric motors can produce very quick and precise torques compared to conventional internal combustion engines. In [1], traction control based on a maximum transmission torque estimation (MTTE) approach was proposed. The estimation was carried out by an open-loop disturbance observer, which required only the input torque and the wheel speed. The estimated maximum transmission torque was used in the control law as a constraint to prevent the slip. Experimental results illustrated the effectiveness and practicality of the proposed control design. The MTTE approach was extended by replacing the open-loop observer with a closed-loop observer in [2]. By doing this, the robustness of the control system was markedly enhanced. In [3], traction control of electric vehicles using a sliding-mode observer to estimate the maximum friction was presented. The observer was based on the LuGre friction model. The controller used the estimated maximum friction to determine the suited maximum torque for the wheels.

Sliding-mode control has been extensively used for control of uncertain nonlinear systems because of its robustness property. The essence of the sliding-mode control is to use a switching control command to drive the controlled system's state trajectory onto a specified sliding surface in the state space and then to keep the state trajectory moving along this surface $[11,12]$.

As far as sliding-mode control of vehicles is concerned, wheel slip control of electric vehicles based on a sliding-mode framework was proposed in [4]. A conditional integrator 
approach was employed to overcome the chattering, enabling a smooth transition to a PI control law when the slip is close to the set point. Experimental results demonstrated a good slip regulation and robustness to disturbances. In [13], a sliding-mode approach to the design of an active braking controller was proposed. The controlled variable was a convex combination of wheel deceleration and wheel slip. The approach offered advantages with respect to pure slip and deceleration control. In [14], a second-order sliding-mode traction force controller for vehicles was proposed. The traction control was achieved by maintaining the wheel slip at a desired value.

This paper presents a robust control scheme for traction control of electric vehicles. The control objective is to operate the vehicles at a desired wheel slip ratio. The paper proposes a simple approach to design a traction controller based on a sliding-mode control framework. The main motivation of the design is the robustness to uncertainties. The implementation of the control design requires tractive forces for feedback, but they are not usually available in practices. To overcome this problem, a PI observer developed in $[15,16]$ is used to estimate the tractive forces. The PI observer has an attractive zero-steady-state feature similar to the well-known PI controllers. This synthesis of the sliding-mode traction controller and the PI observer makes the implementation practical. At the end, the resulting observer-based controller is experimentally validated in a single-wheel test rig.

The rest of the paper is organized as follows. In the next section some preliminaries are provided. The longitudinal dynamic model of the vehicles used in the paper is presented in Section 3, followed by the controller and observer design in Section 4. Simulation results are given in Section 5. In Section 6 an experimental study is presented. The conclusions of this paper are drawn in Section 7.

\section{Preliminaries}

2.1. Sliding-Mode Control. Sliding-mode control based on the equivalent control method is summarized in this subsection. The reader is referred to $[11,12]$ for more details of the method.

The controlled system is expressed as

$$
\dot{x}=f(x)+g(x) u
$$

where $x \in R^{n}$ is the state variable vector, $u \in R^{m}$ is the input vector, and $f(\cdot)$ and $g(\cdot)$ are nonlinear functions. Let $S(x)$ be a desired sliding surface, which is usually chosen according to the control objective. Based on the equivalent control method, the control input vector is written as

$$
u=u_{\mathrm{eq}}+u_{\mathrm{sw}}
$$

where $u_{\text {eq }}$ and $u_{\mathrm{sw}}$ are called the equivalent control and the switching control, respectively. The equivalent control $u_{\text {eq }}$ is determined based on the assumption that the system trajectory is staying on the sliding surface. Thus, it is simply obtained by setting

$$
\dot{S}=0
$$

The switching control $u_{\mathrm{sw}}$ is designed to guarantee that the system trajectory moves towards the sliding surface and stays on it. It is determined such that the reachability condition

$$
S \dot{S}=-\eta|S|, \quad \eta>0
$$

is satisfied.

2.2. Nonlinearity Observer. The nonlinearity observer developed in $[15,16]$ is provided in this subsection. Consider the following nonlinear system:

$$
\begin{gathered}
\dot{x}=A x+N \alpha(t)+M \sigma(t)+B u, \\
y=C x,
\end{gathered}
$$

where $x \in R^{n}$ is the state variable vector, $u \in R^{m}$ is the control vector, $y \in R^{p}$ is the output vector, $A$ is the system matrix, $B$ is the control input matrix, $C$ is the output matrix, and $M$ and $N$ are the constant matrices. Here, $\alpha(t)$ is an unknown nonlinear function whereas $\sigma(t)$ is a known function. The observer is designed to estimate $\alpha(t)$.

The fundamental idea of the observer is to approximate $\alpha(t)$ by a fictitious system:

$$
\begin{aligned}
& \alpha(t) \approx H v(t), \\
& \dot{v}(t)=V v(t) .
\end{aligned}
$$

By substituting (6) into (5), the system can be expressed as

$$
\left[\begin{array}{c}
\dot{x} \\
\dot{v}
\end{array}\right]=\left[\begin{array}{cc}
A & N H \\
0 & V
\end{array}\right]\left[\begin{array}{l}
x \\
v
\end{array}\right]+\left[\begin{array}{cc}
B & M \\
0 & 0
\end{array}\right]\left[\begin{array}{c}
u \\
\sigma
\end{array}\right] .
$$

Thus, the observer is chosen as

$$
\left[\begin{array}{c}
\dot{\hat{x}} \\
\dot{\hat{v}}
\end{array}\right]=\left[\begin{array}{cc}
A & N H \\
0 & V
\end{array}\right]\left[\begin{array}{c}
\hat{x} \\
\widehat{v}
\end{array}\right]+\left[\begin{array}{cc}
B & M \\
0 & 0
\end{array}\right]\left[\begin{array}{l}
u \\
\sigma
\end{array}\right]+\left[\begin{array}{l}
L_{x} \\
L_{v}
\end{array}\right](y-C \hat{x}),
$$

where $L_{x}$ and $L_{v}$ are the observer gain matrices that must be chosen such that the observer is asymptotically stable.

In a special case when $H=I$ and $V=0$ are chosen, the observer is reduced to the proportional-integral (PI) observer:

$$
\begin{gathered}
\dot{\hat{x}}=A \widehat{x}+B u+M \sigma+L_{x}(y-C \widehat{x})+N L_{v} \int(y-C \widehat{x}) d t \\
\widehat{v}=L_{v} \int(y-C \widehat{x}) d t
\end{gathered}
$$

and the estimated nonlinearity is given by

$$
\widehat{\alpha}(t)=\widehat{v}(t) .
$$

The PI observer has been successfully applied to control problems $[17,18]$. The reader is referred to [16] for a proof of the estimation convergence and an analysis of the estimation errors. 


\section{Longitudinal Dynamic Model}

A longitudinal dynamic model of two-axle vehicles is presented. The model is known as a bicycle model, which has three degrees of freedom. The model can be found in [14] and is given as

Vehicle body: $F_{f}+F_{r}-F_{\text {loss }}=m \dot{V}_{x}$,

Front axle: $T_{f}-r_{f} F_{f}=I_{f} \dot{\omega}_{f}$,

Rear axle: $T_{r}-r_{r} F_{r}=I_{r} \dot{\omega}_{r}$,

where $V_{x}$ is the longitudinal velocity of the vehicle center of gravity, $\omega_{f}, \omega_{r}$ are the tire rotational speeds (the subscripts $f$ and $r$ stand for the front and rear axle, resp.), $m$ is the vehicle mass, $I_{f}, I_{r}$ are the moments of inertia, $r_{f}, r_{r}$ are the tire effective rolling radius, $T_{f}, T_{r}$ are the input torques, $F_{f}, F_{r}$ are the tractive forces, $F_{\text {loss }}=c_{x} V_{x}^{2} \cdot \operatorname{sgn}\left(V_{x}\right)+f_{\text {roll }} m g$ combining the aerodynamic drag and the rolling resistance, and $c_{x}$ and $f_{\text {roll }}$ are the aerodynamic drag and rolling resistance coefficients, respectively.

The tractive forces are given by

$$
F_{i}=\mu\left(\lambda_{i}\right) N_{i}, \quad i=\{f, r\},
$$

where $\mu\left(\lambda_{i}\right)$ is the friction coefficient function, $\lambda_{i}$ is the wheel slip ratio defined as

$$
\lambda_{i}= \begin{cases}\frac{r_{i} \omega_{i}-V_{x}}{r_{i} \omega_{i}}, & \text { for driving, } \\ \frac{V_{x}-r_{i} \omega_{i}}{V_{x}}, & \text { for braking }\end{cases}
$$

and $N_{f}, N_{r}$ are the normal loads given as

$$
\begin{aligned}
& N_{f}=\frac{l_{r} m g-l_{h} m \dot{V}_{x}}{l_{f}+l_{r}}, \\
& N_{r}=\frac{l_{f} m g+l_{h} m \dot{V}_{x}}{l_{f}+l_{r}},
\end{aligned}
$$

where $l_{f}$ is the distance from the front axle to the center of gravity, $l_{r}$ is the distance from the rear axle to the center of gravity, and $l_{h}$ is the height of the center of gravity (see Figure 1).

The friction coefficient depends on many factors: tire type, road surface, road condition, wheel slip, and so forth. This makes behaviors of the tractive forces complicated. The friction coefficient usually has to be measured experimentally. The typical friction coefficient in dependency of the slip ratio is shown in Figure 2. It illustrates that some amount of slip is necessary to produce the tractive force; on the other hand, an excessive slip leads to a loss of the force.

\section{Control Design}

4.1. Sliding-Mode Controller Design. A sliding-mode controller is designed for the system described by (11)-(14). The

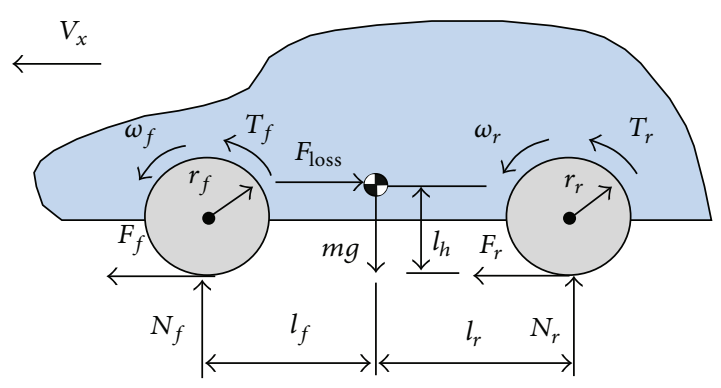

FIGURE 1: Longitudinal model of vehicles.

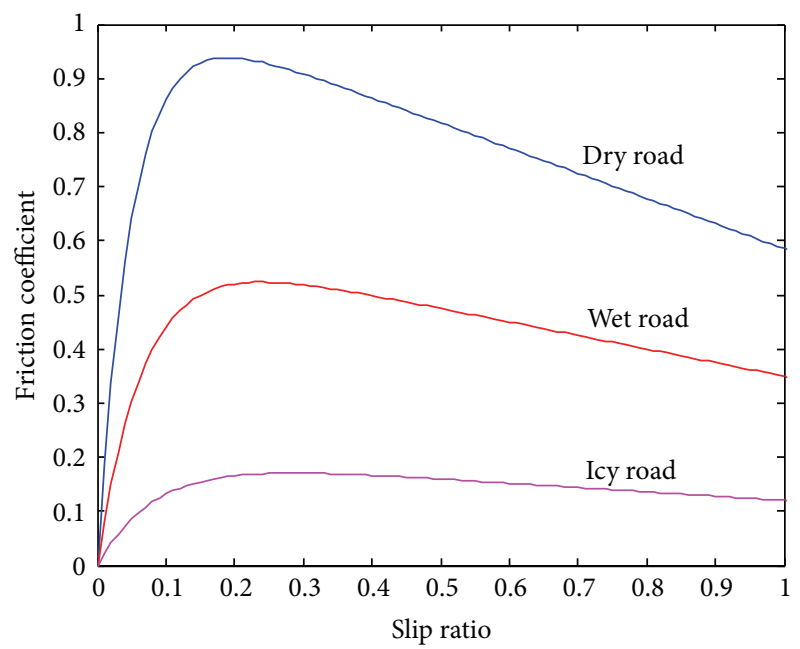

FIgURE 2: Typical trends of longitudinal friction coefficient.

control objective is to drive the vehicle such that the desired slip ratio $\lambda^{*}$ is achieved. First, define the sliding surface as

$$
S_{i}\left(\lambda_{i}, \omega_{i}\right)=\left(\lambda_{i}-\lambda^{*}\right) \omega_{i}, \quad i=\{f, r\}, \omega_{i} \neq 0 .
$$

Taking derivative of the sliding surface yields

$$
\begin{aligned}
\dot{S}_{i}\left(\lambda_{i}, V_{x}\right)= & \frac{F_{\text {loss }}}{r_{i} m}-\frac{1}{r_{i} m} F_{f}-\frac{1}{r_{i} m} F_{r} \\
& -\left(1-\lambda^{*}\right) \frac{r_{i}}{I_{i}} F_{i}+\left(1-\lambda^{*}\right) \frac{1}{I_{i}} u_{i},
\end{aligned}
$$

where $u_{i}=T_{i}, i=\{f, r\}$. Assuming $\dot{S}_{i}=0$ in (16) results in

$$
\begin{aligned}
u_{i, \mathrm{eq}}= & \left(\frac{I_{i}}{1-\lambda^{*}}\right) \\
& \times\left(-\frac{F_{\text {loss }}}{r_{i} m}+\frac{1}{r_{i} m} F_{f}+\frac{1}{r_{i} m} F_{r}+\left(1-\lambda^{*}\right) \frac{r_{i}}{I_{i}} F_{i}\right) .
\end{aligned}
$$

Next, letting $u_{i}=u_{i, \mathrm{eq}}+u_{i, \mathrm{sw}}$ and utilizing the reachability condition (4) result in

$$
u_{i, \mathrm{sw}}=-\eta_{i}\left(\frac{I_{i}}{1-\lambda^{*}}\right) \operatorname{sgn}\left(S_{i}\right), \quad \eta_{i}>0,
$$


where

$$
\operatorname{sgn}\left(S_{i}\right)=\frac{\left|S_{i}\right|}{S_{i}}= \begin{cases}1, & S_{i}>0 \\ 0, & S_{i}=0 \\ -1, & S_{i}<0\end{cases}
$$

From (17) and (18), the sliding-mode control law can be concluded as

$$
\begin{aligned}
u_{i}=\left(\frac{I_{i}}{1-\lambda^{*}}\right)(- & \frac{F_{\text {loss }}}{r_{i} m}+\frac{1}{r_{i} m} F_{f}+\frac{1}{r_{i} m} F_{r} \\
& \left.+\left(1-\lambda^{*}\right) \frac{r_{i}}{I_{i}} F_{i}-\eta_{i} \operatorname{sgn}\left(S_{i}\right)\right) .
\end{aligned}
$$

Note that the control law requires $F_{f}, F_{r}$ for feedback, but they are usually not available. Thus, an observer is needed to estimate $F_{f}, F_{r}$.

4.2. Nonlinearity Observer Design. A nonlinearity observer is designed to estimate $F_{f}, F_{r}$. First, express (11) in the form (5) as

$$
\begin{aligned}
\dot{x}= & {\left[\begin{array}{lll}
0 & 0 & 0 \\
0 & 0 & 0 \\
0 & 0 & 0
\end{array}\right] x+\left[\begin{array}{cc}
\frac{1}{m} & \frac{1}{m} \\
-\frac{r_{f}}{I_{f}} & 0 \\
0 & -\frac{r_{r}}{I_{r}}
\end{array}\right] \alpha(t) } \\
& +\left[\begin{array}{cc}
\frac{1}{m} \\
0 \\
0
\end{array}\right] \sigma(t)+\left[\begin{array}{cc}
0 & 0 \\
\frac{1}{I_{f}} & 0 \\
0 & \frac{1}{I_{r}}
\end{array}\right] u, \\
y= & {\left[\begin{array}{lll}
1 & 0 & 0 \\
0 & 1 & 0 \\
0 & 0 & 1
\end{array}\right] x, }
\end{aligned}
$$

where $x^{T}=\left[\begin{array}{lll}V_{x} & \omega_{f} & \omega_{r}\end{array}\right], u^{T}=\left[\begin{array}{ll}T_{f} & T_{r}\end{array}\right], \alpha(t)^{T}=\left[\begin{array}{ll}F_{f} & F_{r}\end{array}\right]$, and $\sigma(t)=F_{\text {loss }}$. Consequently, the observer (8) with $H=I$ and $V=0$ can be expressed as

$$
\begin{aligned}
\dot{\vec{X}}= & {\left[\begin{array}{ccccc}
0 & 0 & 0 & \frac{1}{m} & \frac{1}{m} \\
0 & 0 & 0 & -\frac{r_{f}}{I_{f}} & 0 \\
0 & 0 & 0 & 0 & -\frac{r_{r}}{I_{r}} \\
0 & 0 & 0 & 0 & 0 \\
0 & 0 & 0 & 0 & 0
\end{array}\right] \widehat{X}+\left[\begin{array}{ccc}
0 & 0 & \frac{1}{m} \\
\frac{1}{I_{f}} & 0 & 0 \\
0 & \frac{1}{I_{r}} & 0 \\
0 & 0 & 0 \\
0 & 0 & 0
\end{array}\right] U } \\
& +L\left(y-\left[\begin{array}{ccccc}
1 & 0 & 0 & 0 & 0 \\
0 & 1 & 0 & 0 & 0 \\
0 & 0 & 1 & 0 & 0
\end{array}\right] \widehat{X}\right),
\end{aligned}
$$

where $\widehat{X}^{T}=\left[\begin{array}{lllll}\widehat{V}_{x} & \widehat{\omega}_{f} & \widehat{\omega}_{r} & \widehat{F}_{f} & \widehat{F}_{r}\end{array}\right]$ and $U^{T}=\left[\begin{array}{lll}T_{f} & T_{r} & F_{\text {loss }}\end{array}\right]$. Here, $L$ is the observer gain matrix that must be chosen such that $\widehat{X} \rightarrow X$, resulting in $\widehat{F}_{f} \rightarrow F_{f}$ and $\widehat{F}_{r} \rightarrow F_{r}$ as desired.
TABLE 1: Parameters of the vehicle and the friction model.

\begin{tabular}{lc}
\hline Parameter & Value \\
\hline$m$ & $1202 \mathrm{~kg}$ \\
$I_{f}, I_{r}$ & $1.07 \mathrm{kgm}^{2}$ \\
$l_{f}$ & $1.15 \mathrm{~m}$ \\
$l_{r}$ & $1.45 \mathrm{~m}$ \\
$l_{h}$ & $0.53 \mathrm{~m}$ \\
$c_{x}$ & 0.4 \\
$f_{\text {roll }}$ & 0.013 \\
$r_{f}, r_{r}$ & $0.32 \mathrm{~m}$ \\
$C_{1}$ & 1.05 \\
$C_{2}$ & 20.02 \\
$C_{3}$ & 0.4646 \\
\hline
\end{tabular}

\section{Numerical Studies and Results}

The vehicle model in (11)-(14) is used as the control plant. The vehicle parameters are adopted from [14] and are summarized in Table 1. The friction coefficient function used in this paper is the Burckhardt friction model [9]:

$$
\mu(\lambda)=C_{1}\left(1-e^{-C_{2} \lambda}\right)-C_{3} \lambda,
$$

where $C_{1}, C_{2}$, and $C_{3}$ are model parameters. The values of the friction model are also listed in Table 1 . This friction model yields the maximum friction coefficient at $\lambda \approx 0.2$.

5.1. Sliding-Mode Controller. The control law (20) is used. Here, it is assumed that $F_{f}, F_{r}$ are available for feedback. This assumption will be removed later in Section 5.3. The tuning parameters $\eta_{f}=\eta_{r}=120$ are chosen. To achieve the maximum tractive forces, the desired slip ratio $\lambda^{*}$ is set to 0.2. The results are shown in Figures $3-5$. Note that, at time $=$ $20 \mathrm{sec}$, a ten-time decrease of the friction coefficient was introduced to simulate a change in road condition. Figure 3 shows that the sliding-mode controller successfully drove the system to operate at the desired wheel slip ratio.

Time histories of the input torques are presented in Figure 4. It is seen from this figure that the controller effectively reduced the input torques to keep the wheel slip ratios constant when the friction coefficient was decreased. Figure 5 displays time histories of the vehicle velocity and the tire rotational speeds. It shows that the velocity and the rotational speeds decreased corresponding to the input torques.

5.2. Nonlinearity Observer. The observer (22) is used. The observer gain $L$ is determined through the pole-placement technique. The desired poles are simply chosen to be $-1,-2$, $-3,-4$, and -5 and the following gain matrix is obtained:

$$
L=\left[\begin{array}{ccc}
3.041 & -0.079 & 0.128 \\
-0.239 & 6.545 & 0.911 \\
0.241 & 0.664 & 5.414 \\
1.853 & -30.11 & -8.293 \\
-0.715 & -5.375 & -16.21
\end{array}\right]
$$



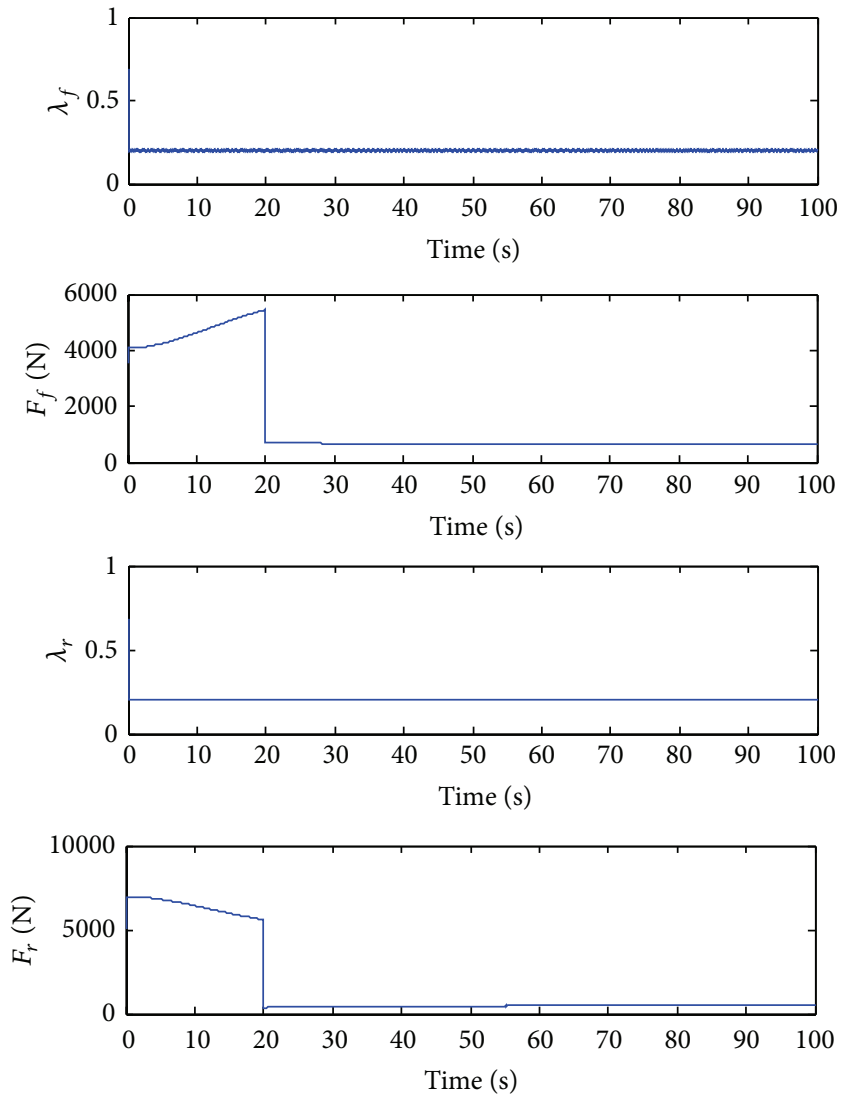

FIGURE 3: Wheel slip ratios and tire tractive forces.
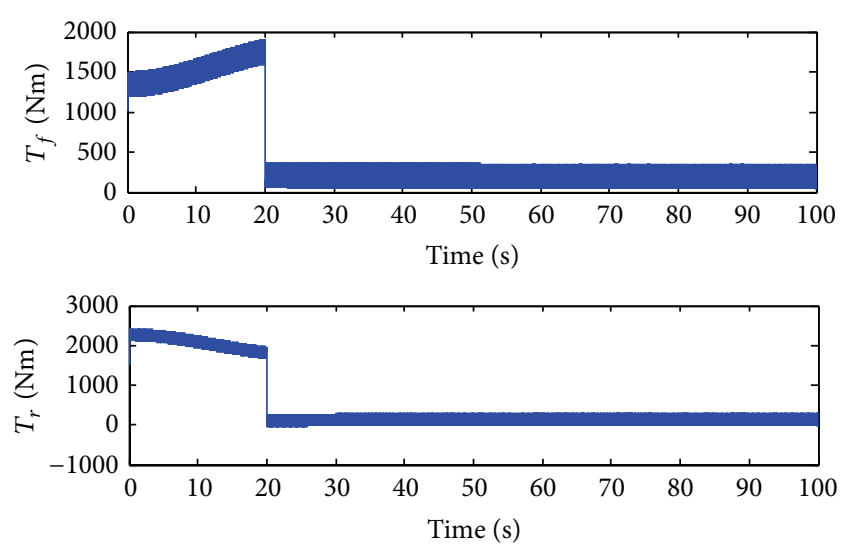

FIGURE 4: Input torques.

The results are shown in Figures 6-8. The input torques $T_{f}, T_{r}$ were initially set to $500 \mathrm{Nm}$ and then stepped up to $1500 \mathrm{Nm}$ at time $=200 \mathrm{sec}$. The results show that the observer correctly estimated all state variables including the tractive forces.

Comparisons of true and estimated velocity and rotational speeds are presented in Figure 6. It is seen from the figure that there are overshoots in the estimated rotational speeds when the step change of the input torques occurred. However, they were quickly converged back to the true values. The estimations of the tractive forces are presented in Figures
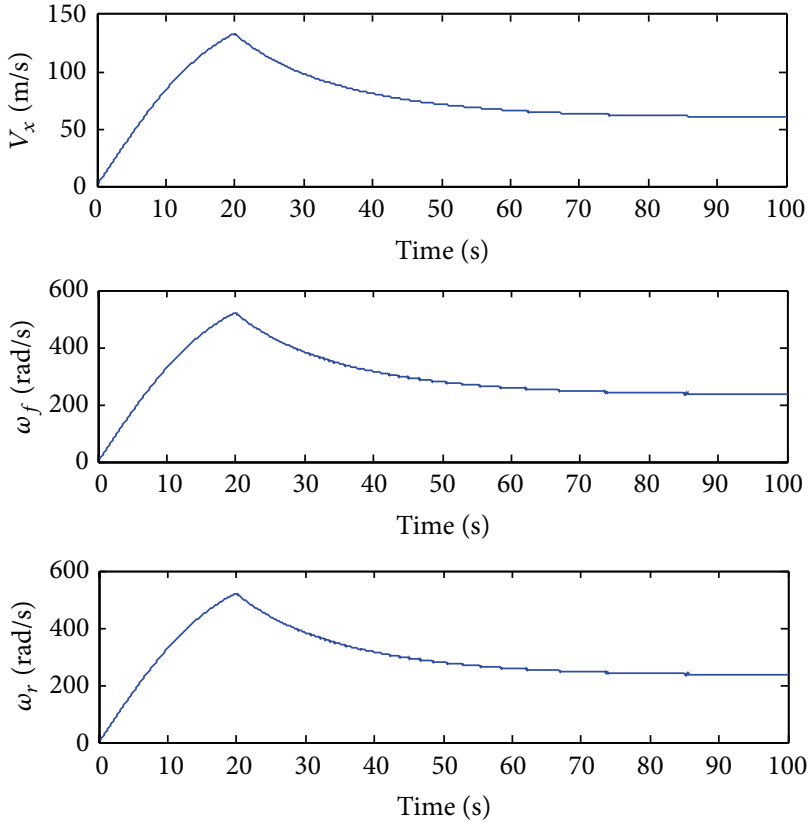

FIGURE 5: Velocity and rotational speeds.
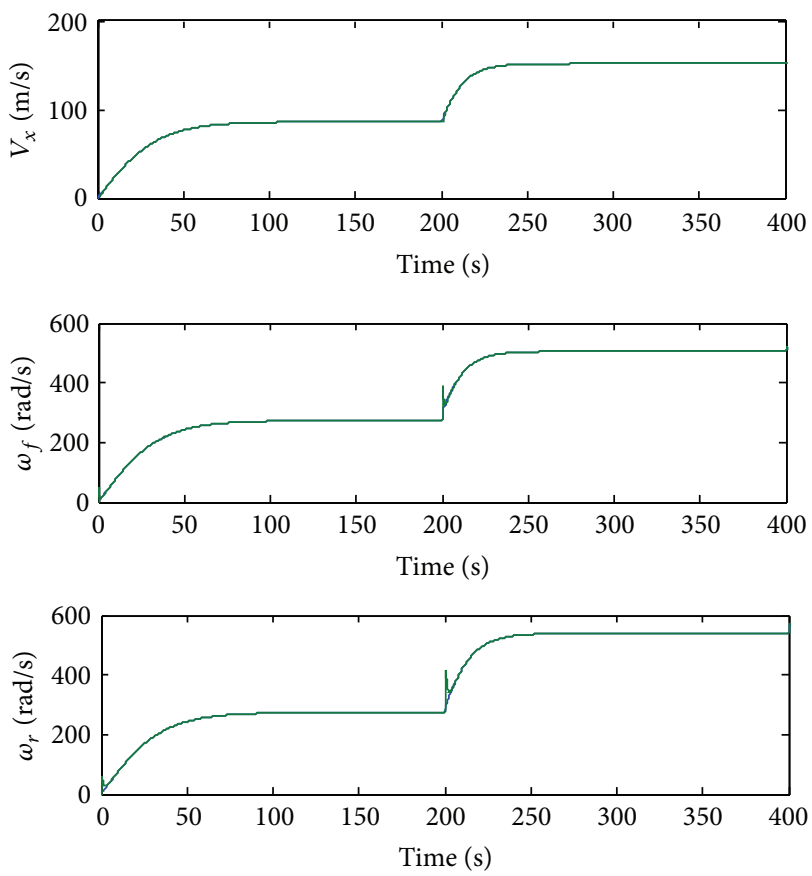

FIGURE 6: Comparison of true and estimated velocity and rotational speeds.

7 and 8. In Figure 8, it is seen that the estimations converged to the true values within 3 and $6 \mathrm{sec}$ for the front and rear wheels, respectively.

Moreover, to investigate the measurement noises effects on the accuracy of the estimations, we added zero-mean normal distribution noises with the variance of 50 into all measurements. We found that the observer still worked well. The results similar to those in Figures 6-8 were obtained. 

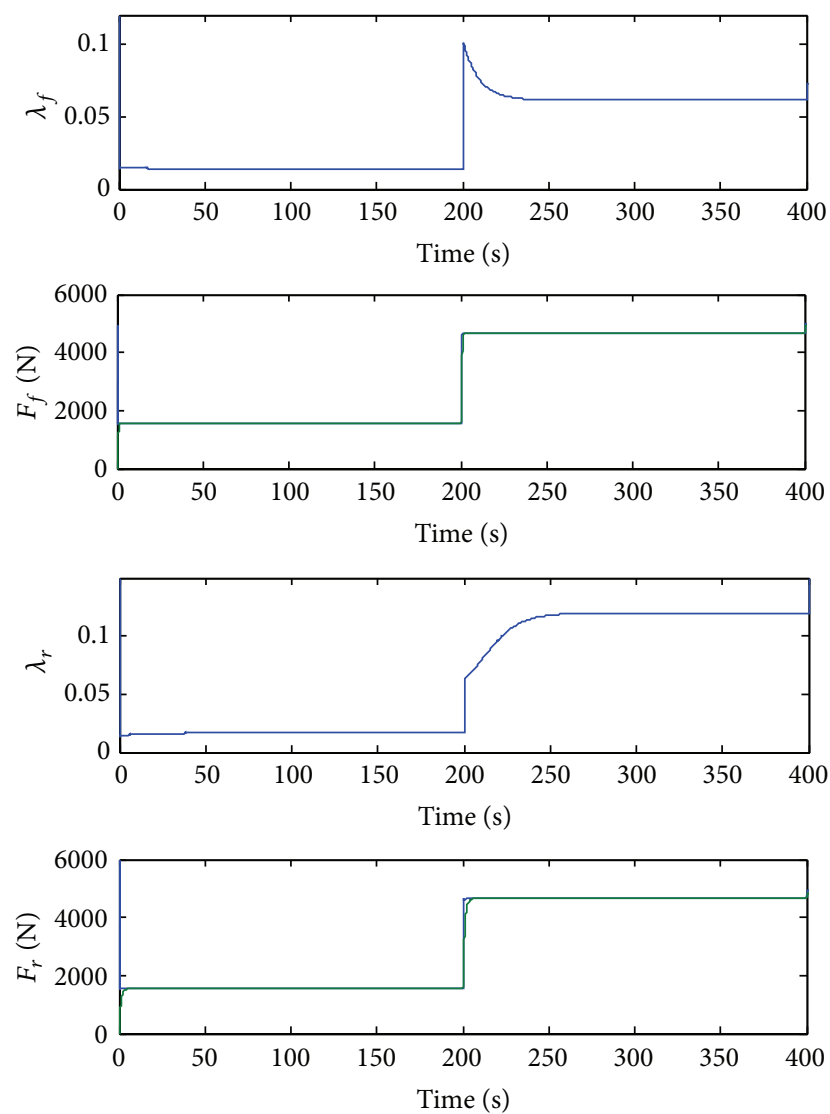

Figure 7: Wheel slip ratios and comparison of true and estimated tractive forces.
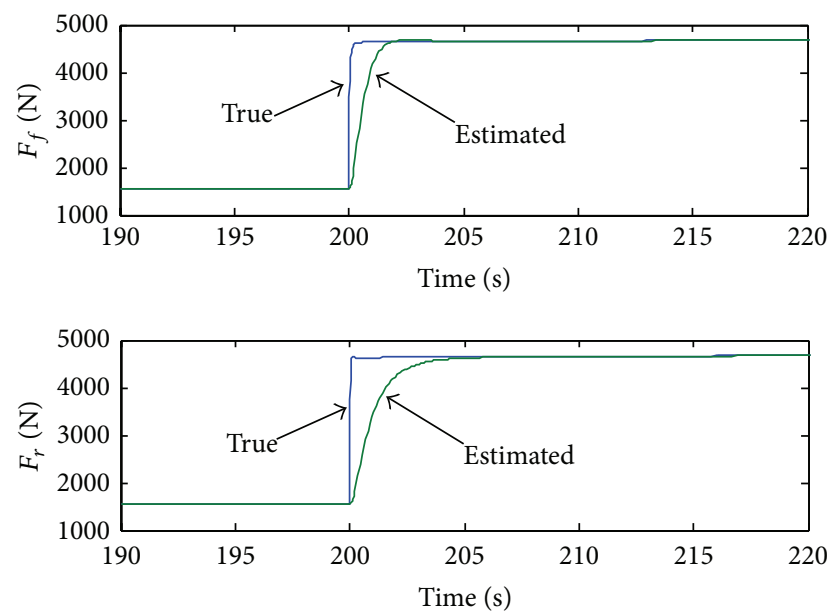

Figure 8: Comparison of true and estimated tractive forces around time $=200 \mathrm{sec}$.

Figure 9 displays an example of the results. Note that there are some small oscillations in the estimations due to the noises.

5.3. Sliding-Mode Controller with Nonlinearity Observer. In this subsection, the scenario in Section 5.1 is resimulated without the availability assumption of $F_{f}, F_{r}$. The observer presented in Section 5.2 is used to estimate $F_{f}, F_{r}$. The results are shown in Figures 10-12.
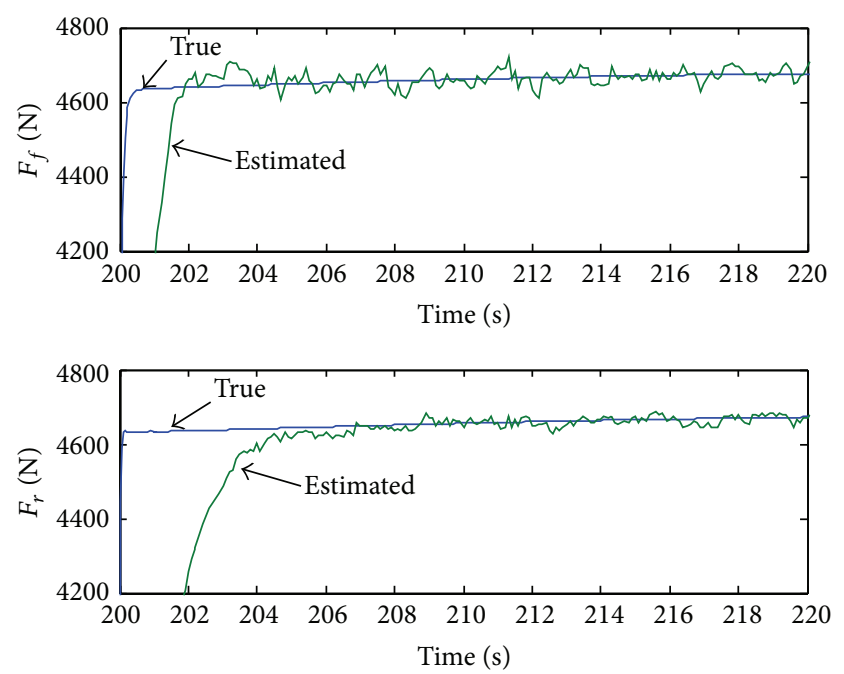

FIGURE 9: Comparison of true and estimated tractive forces when the measurements degraded by noises.
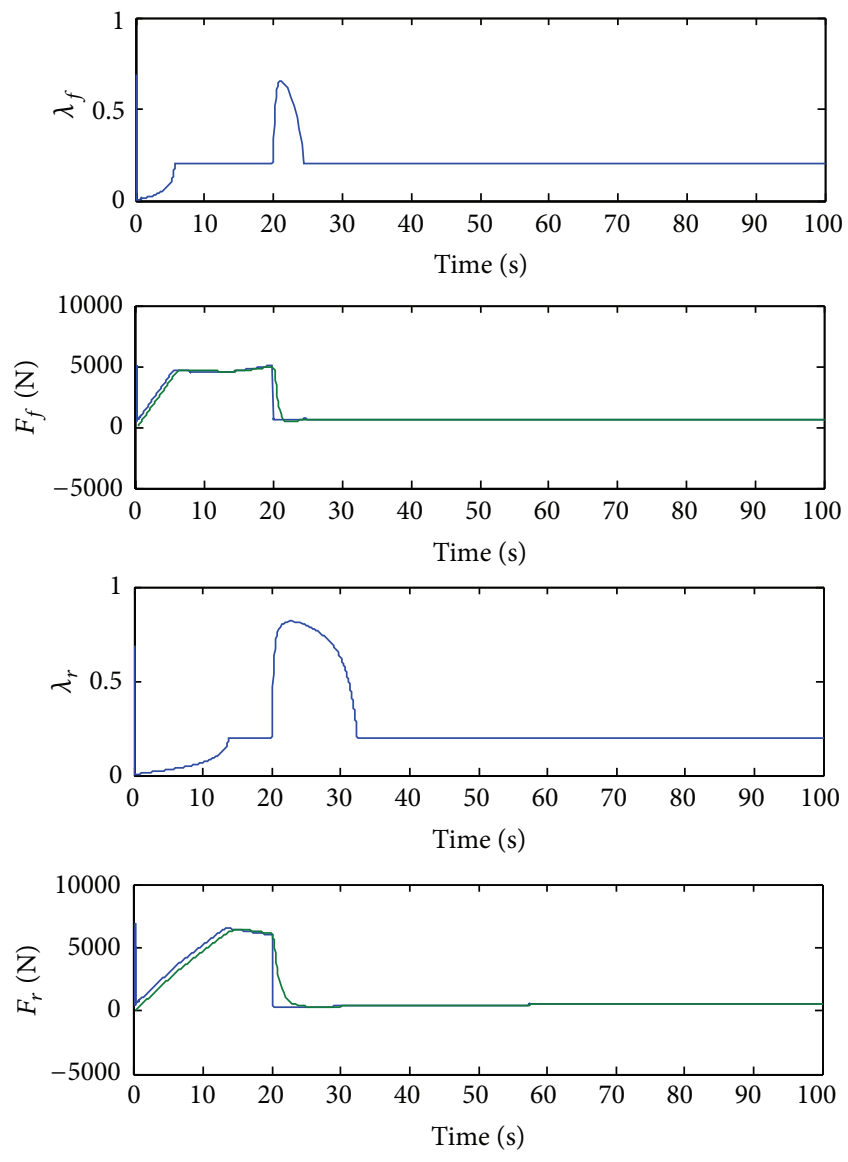

FIGURE 10: Wheel slip ratios and tire tractive forces using the observer-based controller.

Figure 10 shows that the observer-based controller was able to drive the system to operate at the desired wheel slip ratio. Note that there are jumps of the slip ratios occurring when the friction coefficient was decreased, but they asymptotically converged back to the desired value. The jumps are due to the dynamics of the observer. 

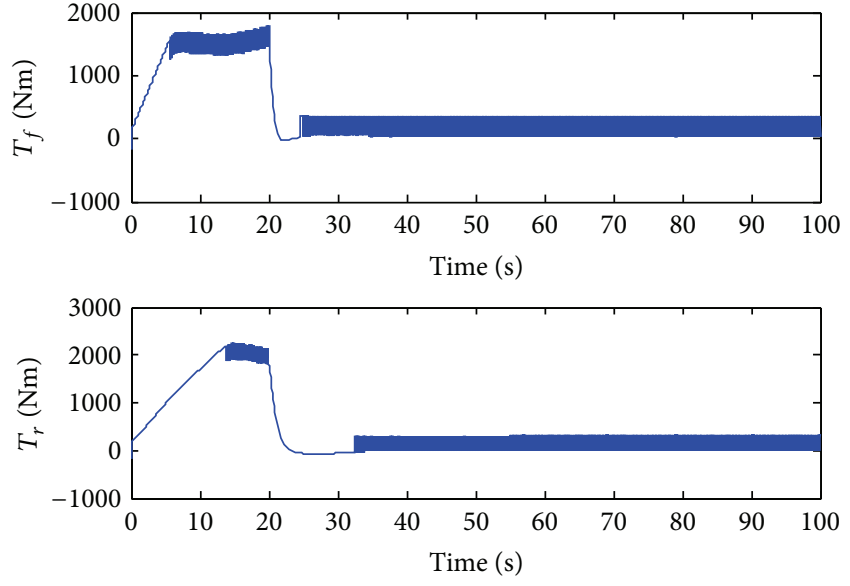

FIGURE 11: Input torques using the observer-based controller.
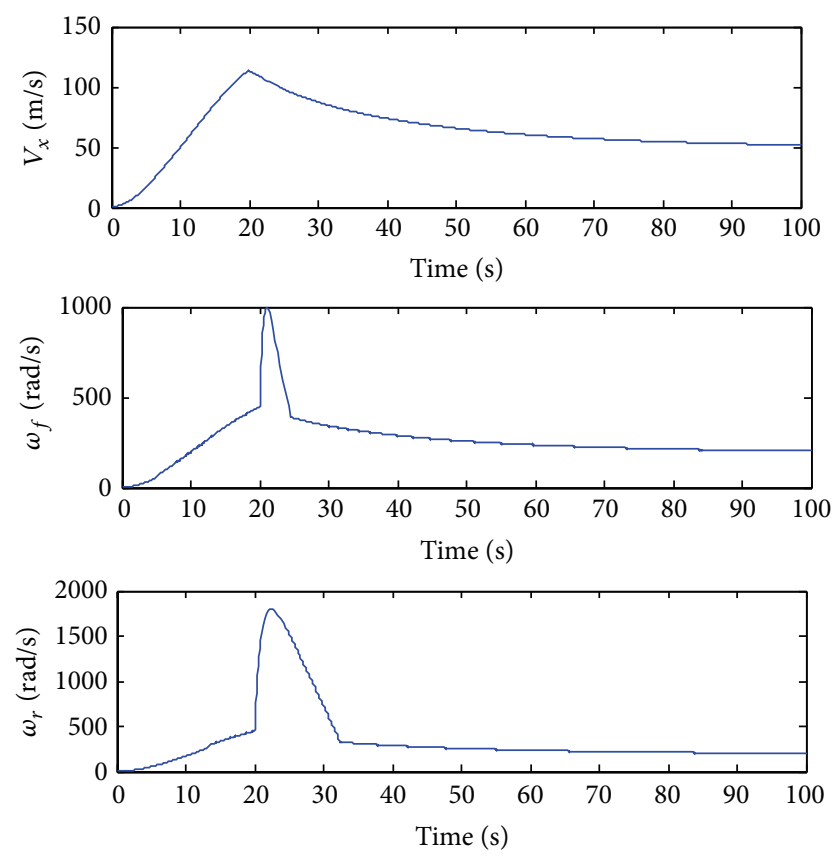

FIGURE 12: Velocity and rotational speeds using the observer-based controller.

Time histories of the input torques are presented in Figure 11. Similar to the results in Figure 4, the controller effectively reduced the input torques to keep the wheel slip ratios constant. Figure 12 displays time histories of the vehicle velocity and the tire rotational speeds. It is observed that there were large overshoots in the rotational speeds when the friction was decreased. These overshoots are due to the dynamics of the observer.

\section{Experimental Studies and Results}

The experimental studies are carried on a single-wheel test rig (see Figure 13). The test rig simulates the longitudinal dynamic of electric vehicles. It consists of a drum set, a wheel

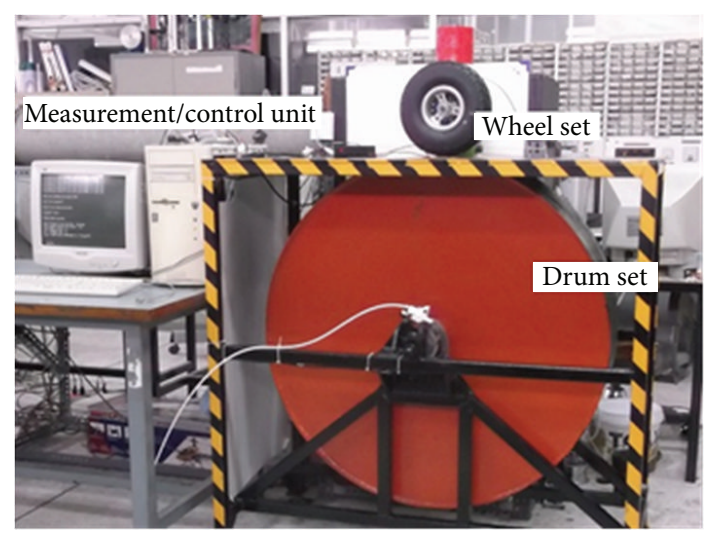

FIGURE 13: Photograph of the single-wheel test rig.

set, and a measurement/control unit. The role of the drum set is to let the wheel behave like rolling on a road. The diameter and width of the drum are approximately 1.0 and 0.3 meters, respectively. The drum set is also equipped with a rotational speed sensor. The wheel set consists of a tire, a 4-inch-rim wheel, a brushed DC motor, and loading masses. The wheel is directly driven by the motor through a rigid shaft. The wheel set is also equipped with another rotational speed sensor. The measurement/control unit consists of a signal condition circuit and a PC computer. The computer is installed with a 12-bit analog/digital interface board. The two rotational speed signals are fed back to the computer through the interface board. The control sampling period is $0.5 \mathrm{sec}$.

A mathematical model of the test rig can be summarized:

$$
\begin{aligned}
& \text { Drum: } r_{d}^{2} F-c_{d} V_{x}=I_{d} \dot{V}_{x}, \\
& \text { Wheel: } b V_{\text {in }}-r_{w} F-a \omega=I_{w} \dot{\omega},
\end{aligned}
$$

where $V_{x}=\Omega r_{d}$ is the simulated longitudinal velocity, $\Omega$ is the drum rotational speed, $r_{d}$ is the drum radius, $\omega$ is the wheel rotational speed, $V_{\text {in }}$ is the motor voltage input, $I_{d}, I_{w}$ are the moments of inertia (the subscripts $d$ and $w$ stand for the drum and wheel, resp.), $r_{w}$ is the wheel radius, $a=$ $\left(k_{T} k_{b} / R+c_{w}\right)$ and $b=\left(k_{T} / R\right) K_{0}$ are constants, $k_{T}$ is the motor torque constant, $k_{b}$ is the motor back-EMF constant, $R$ is the motor resistance, $c_{d}, c_{w}$ are the damping constants, $K_{0}$ is the gain of the motor-drive unit, and $F$ is the tractive force. The parameters of the test rig are summarized in Table 2.

6.1. Control Design. First, a sliding-mode controller is designed. Define the sliding surfaces as

$$
s\left(\lambda, \omega_{i}\right)=\left(\lambda-\lambda^{*}\right) \omega_{i}, \quad \omega_{i} \neq 0 .
$$

Taking derivative of the sliding surface yields

$$
\begin{aligned}
\dot{s}\left(\lambda, V_{x}\right)= & \frac{c_{d}}{I_{d} r_{w}} V_{x}-\frac{r_{d}^{2}}{I_{d} r_{w}} F_{\mu}-\left(1-\lambda^{*}\right) \frac{a}{I_{w}} \omega \\
& -\left(1-\lambda^{*}\right) \frac{r_{w}}{I_{w}} F_{\mu}+\left(1-\lambda^{*}\right) \frac{b}{I_{w}} u,
\end{aligned}
$$


TABLE 2: Parameters of the experimental test rig.

\begin{tabular}{lc}
\hline Parameter & Value \\
\hline$I_{d}$ & $24.95 \mathrm{kgm}^{2}$ \\
$I_{w}$ & $0.0098 \mathrm{kgm}^{2}$ \\
$r_{d}$ & $0.5 \mathrm{~m}$ \\
$r_{w}$ & $0.13125 \mathrm{~m}$ \\
$a$ & 0.0392 \\
$b$ & 0.5096 \\
$c_{d}$ & $0.05 \mathrm{~N} / \mathrm{ms}^{-1}$ \\
\hline
\end{tabular}

where $u=V_{\text {in }}$. By following the procedure in Section 4.1, the sliding-mode control law can be found as

$$
\begin{aligned}
u=\left(\frac{I_{w}}{\left(1-\lambda^{*}\right) b}\right)( & -\frac{c_{d}}{I_{d} r_{w}} V_{x}+\frac{r_{d}^{2}}{I_{d} r_{w}} F_{\mu}+\left(1-\lambda^{*}\right) \frac{a}{I_{w}} \omega \\
& \left.+\left(1-\lambda^{*}\right) \frac{r_{w}}{I_{w}} F_{\mu}-\eta \operatorname{sgn}(s)\right) .
\end{aligned}
$$

Next, a tractive force observer is designed. Rewrite (25) as

$$
\begin{aligned}
& \dot{x}=\left[\begin{array}{cc}
-\frac{c_{d}}{I_{d}} & 0 \\
0 & -\frac{a}{I_{w}}
\end{array}\right] x+\left[\begin{array}{c}
\frac{r_{d}^{2}}{I_{d}} \\
-\frac{r_{w}}{I_{w}}
\end{array}\right] f(t)+\left[\begin{array}{c}
0 \\
\frac{b}{I_{w}}
\end{array}\right] u, \\
& y=\left[\begin{array}{ll}
1 & 0 \\
0 & 1
\end{array}\right] x,
\end{aligned}
$$

where $x^{T}=\left[\begin{array}{ll}V_{x} & \omega\end{array}\right], u=V_{\text {in }}$, and $f(t)=F_{\mu}$. By following the procedure in Section 4.2, the observer can be concluded as

$$
\begin{aligned}
\dot{\hat{X}}= & {\left[\begin{array}{ccc}
-\frac{c_{d}}{I_{d}} & 0 & \frac{r_{d}^{2}}{I_{d}} \\
0 & -\frac{a}{I_{w}} & -\frac{r_{w}}{I_{w}} \\
0 & 0 & 0
\end{array}\right] \widehat{X} } \\
& +\left[\begin{array}{c}
0 \\
\frac{b}{I_{w}} \\
0
\end{array}\right] u+L\left(y-\left[\begin{array}{lll}
1 & 0 & 0 \\
0 & 1 & 0
\end{array}\right] \widehat{X}\right),
\end{aligned}
$$

where $\widehat{X}^{T}=\left[\begin{array}{lll}\widehat{V}_{x} & \widehat{\omega} & \widehat{F}_{\mu}\end{array}\right]$ and $u=V_{\text {in }}$. The gain matrix $L$ must be chosen such that $\widehat{X} \rightarrow X$.

6.2. Experimental Results. The controller comprises the control law (28) and the observer (30). The parameters in the control law (28) are chosen as $\lambda^{*}=0.2$ and $\eta=10$. The gain matrix $L$ in the observer (30) is designed by simply placing the observer poles at $-1,-2$, and -3 . Since the observer is implemented in a digital computer with the sampling period 0.5 seconds, the corresponding discrete-time desired poles
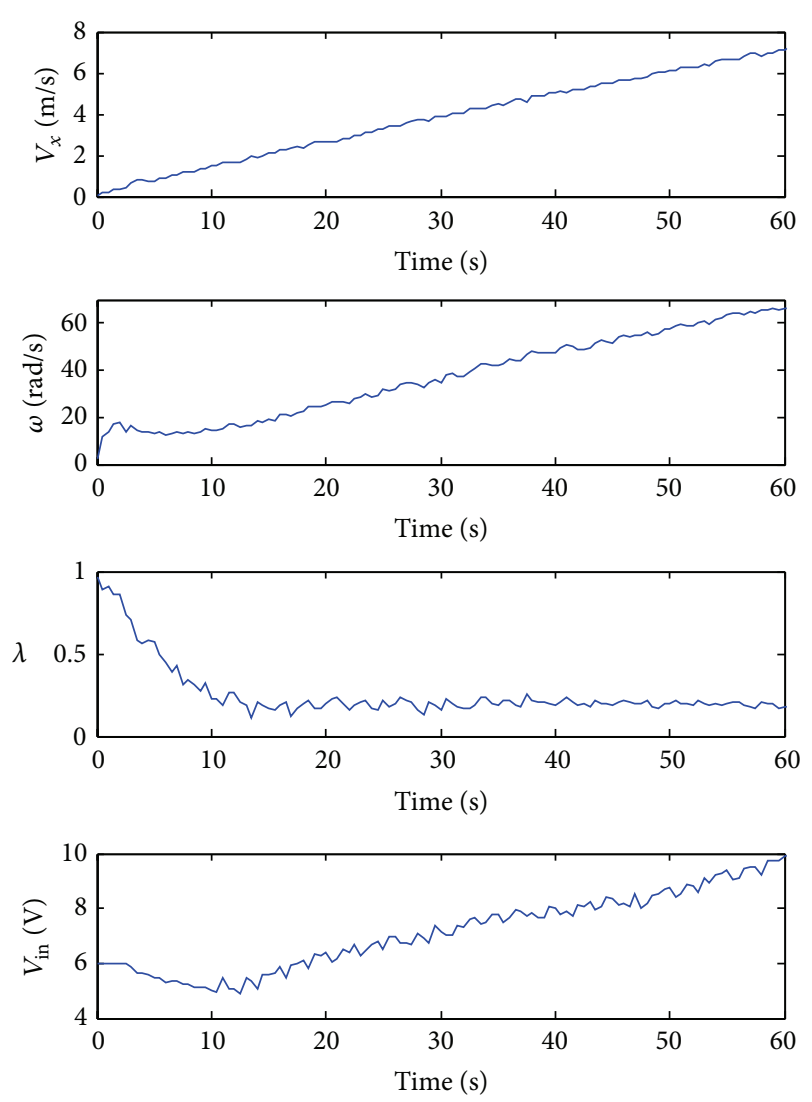

FIGURE 14: Experimental results using the observer-based controller.

become $0.6065,0.3679$, and 0.2231 . The following gain matrix is obtained:

$$
L=\left[\begin{array}{cc}
0.6306 & 0.0006 \\
0.2680 & 0.3062 \\
-0.0591 & -0.1058
\end{array}\right]
$$

Experimental results are shown in Figure 14. Here, $V_{\text {in }}$ was set to 6 volt for the first few seconds prior the activation of controller. The results show that the observer-based controller was able to drive the system to operate at $\lambda=0.2$ successfully.

\section{Conclusions}

This paper has proposed a robust observer-based slidingmode control scheme for a vehicle traction control problem. The sliding-mode controller is designed using an equivalent control method. The control objective is to operate the vehicles at a desired wheel slip ratio. Based on a PI observer design, the observer estimates the tractive forces to be used in the control law. Only a vehicle longitudinal velocity measurement and the tire rotational speed measurements are required in the control loop. However, since the vehicle longitudinal velocity measurement might not be commonly found in vehicles, this requirement is considered as the limitation of the proposed scheme. The simulation and experimental results illustrated the effectiveness of the proposed 
observer-based controller. Last but not least, although the derivation of the sliding-mode control law guarantees the convergence of the desired slip, the coupling of the observer and the controller still does not theoretically confirm the convergence. This theoretical work may be considered as a needed future research.

\section{Conflict of Interests}

The author declares that there is no conflict of interests regarding the publication of this paper.

\section{Acknowledgment}

The author gratefully acknowledges the support provided by Science and Technology Research Institute, King Mongkut's University of Technology North Bangkok.

\section{References}

[1] D. Yin, S. Oh, and Y. Hori, "A novel traction control for EV based on maximum transmissible torque estimation," IEEE Transactions on Industrial Electronics, vol. 56, no. 6, pp. 20862094, 2009.

[2] J.-S. Hu, D. Yin, and Y. Hori, "Fault-tolerant traction control of electric vehicles," Control Engineering Practice, vol. 19, no. 2, pp. 204-213, 2011.

[3] G. A. Magallan, C. H. De Angelo, and G. O. Garcia, "Maximization of the traction forces in a $2 \mathrm{WD}$ electric vehicle," IEEE Transactions on Vehicular Technology, vol. 60, no. 2, pp. 369380, 2011.

[4] R. de Castro, R. E. Araujo, and D. Freitas, "Wheel slip control of EVs based on sliding mode technique with conditional integrators," IEEE Transactions on Industrial Electronics, vol. 60, no. 8, pp. 3256-3271, 2013.

[5] B. Allaoua, B. Mebarki, and A. Laoufi, "A robust fuzzy sliding mode controller synthesis applied on boost DC-DC converter power supply for electric vehicle propulsion system," International Journal of Vehicular Technology, vol. 2013, Article ID 587687, 9 pages, 2013.

[6] K. Nam, H. Fujimoto, and Y. Hori, "Advanced motion control of electric vehicles based on robust lateral tire force control via active front steering," IEEE/ASME Transactions on Mechatronics, vol. 19, no. 1, pp. 289-299, 2014.

[7] V. Sharma and S. Purwar, "Nonlinear controllers for a lightweighted all-electric vehicle using chebyshev neural network," International Journal of Vehicular Technology, vol. 2014, Article ID 867209, 14 pages, 2014.

[8] L. Chen, X. Sun, H. Jiang, and X. Xu, "A high-performance control method of constant $v / f$-controlled induction motor drives for electric vehicles," Mathematical Problems in Engineering, vol. 2014, Article ID 386174, 10 pages, 2014.

[9] U. Kiencke and L. Nielsen, Automotive Control Systems: For Engine, Driveline, and Vehicle, Springer, New York, NY, USA, 2005.

[10] R. Rajamani, Vehicle Dynamics and Control, Springer, New York, NY, USA, 2012.

[11] H. K. Khalil, Nonlinear Systems, Prentice Hall, Upper Saddle River, NJ, USA, 1996.
[12] C. Edwards and S. K. Spurgeon, Sliding Mode Control: Theory and Applications, Taylor \& Francis, London, UK, 1998.

[13] M. Tanelli, R. Sartori, and S. M. Savaresi, "Combining slip and deceleration control for brake-by-wire control systems: a sliding-mode approach," European Journal of Control, vol. 13, no. 6, pp. 593-611, 2007.

[14] M. Amodeo, A. Ferrara, R. Terzaghi, and C. Vecchio, "Wheel slip control via second-order sliding-mode generation," IEEE Transactions on Intelligent Transportation Systems, vol. 11, no. 1, pp. 122-131, 2010.

[15] P. C. Müller, "Estimation and compensation of nonlinearities," in Proceedings of the 1st Asian Control Conference, vol. 2, pp. 641644, Tokyo, Japan, 1994.

[16] P. C. Müller, "Design of PI-observers and compensators for nonlinear control system," in Advances in Mechanics, Dynamics and Control, F. L. Chernousko, G. V. Kostin, and V. V. Saurin, Eds., pp. 223-231, Nauka, Moscow, Russia, 2008.

[17] F. Heidtmann, I. Krajcin, and D. Söffker, "Observer-based control and disturbance compensation of elastic mechanical 2D-/3D-structures," in Proceedings of the 2nd International Conference on Dynamics, Vibration, and Control, pp. 1-4, Beijing, China, 2006.

[18] P. Nakkarat and S. Kuntanapreeda, "Observer-based backstepping force control of an electrohydraulic actuator," Control Engineering Practice, vol. 17, no. 8, pp. 895-902, 2009. 

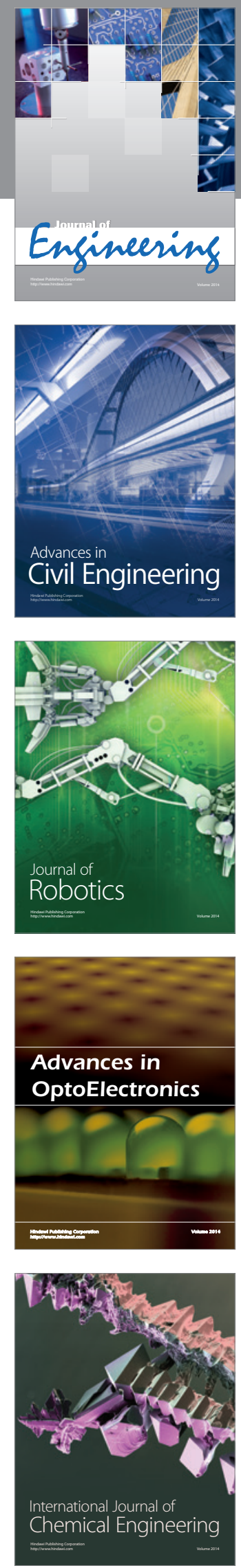

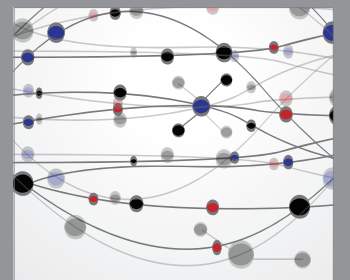

The Scientific World Journal
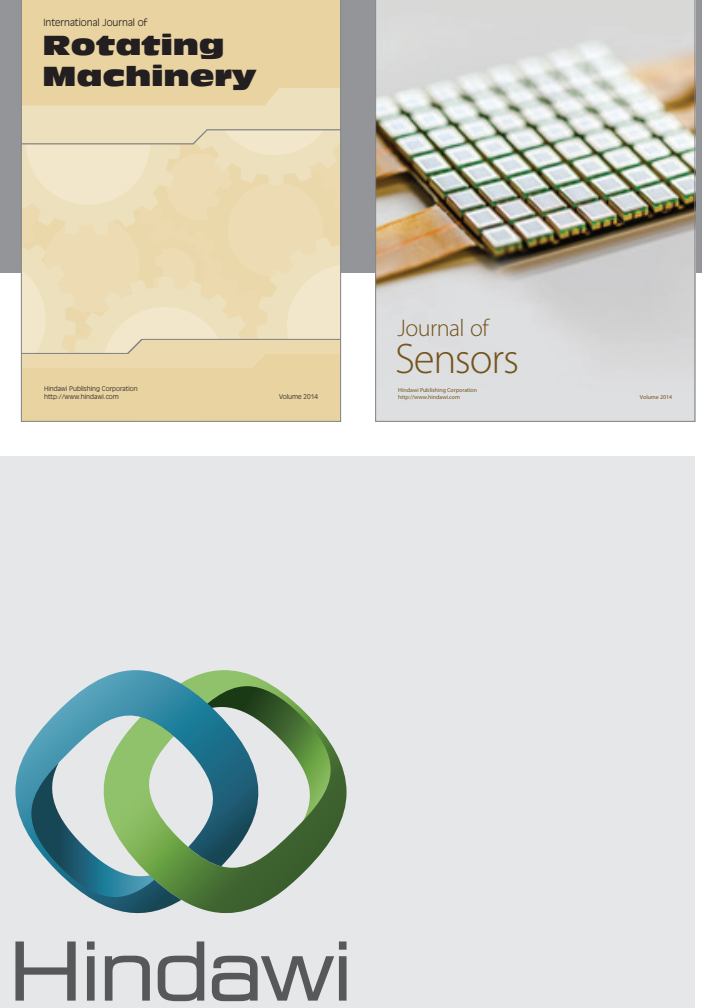

Submit your manuscripts at http://www.hindawi.com
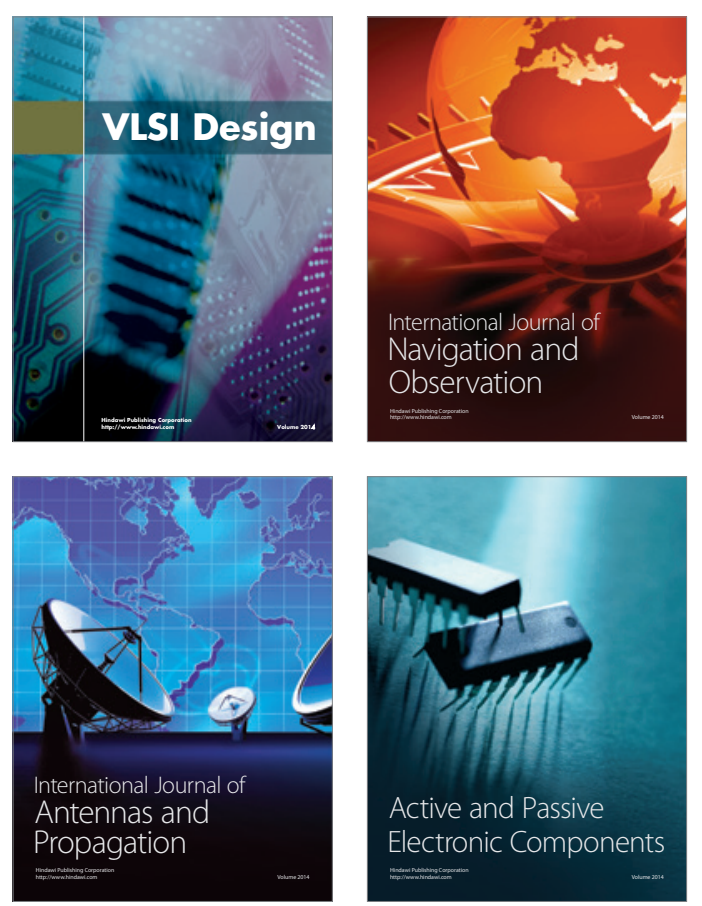
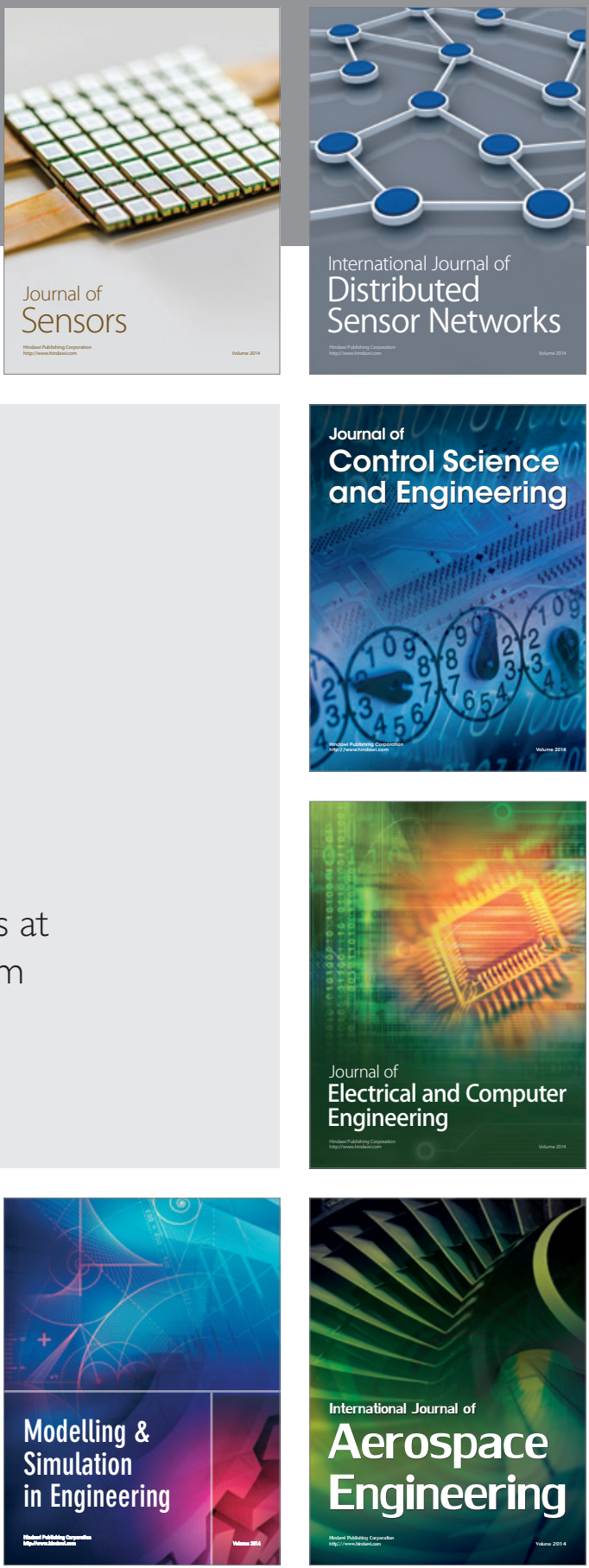

Journal of

Control Science

and Engineering
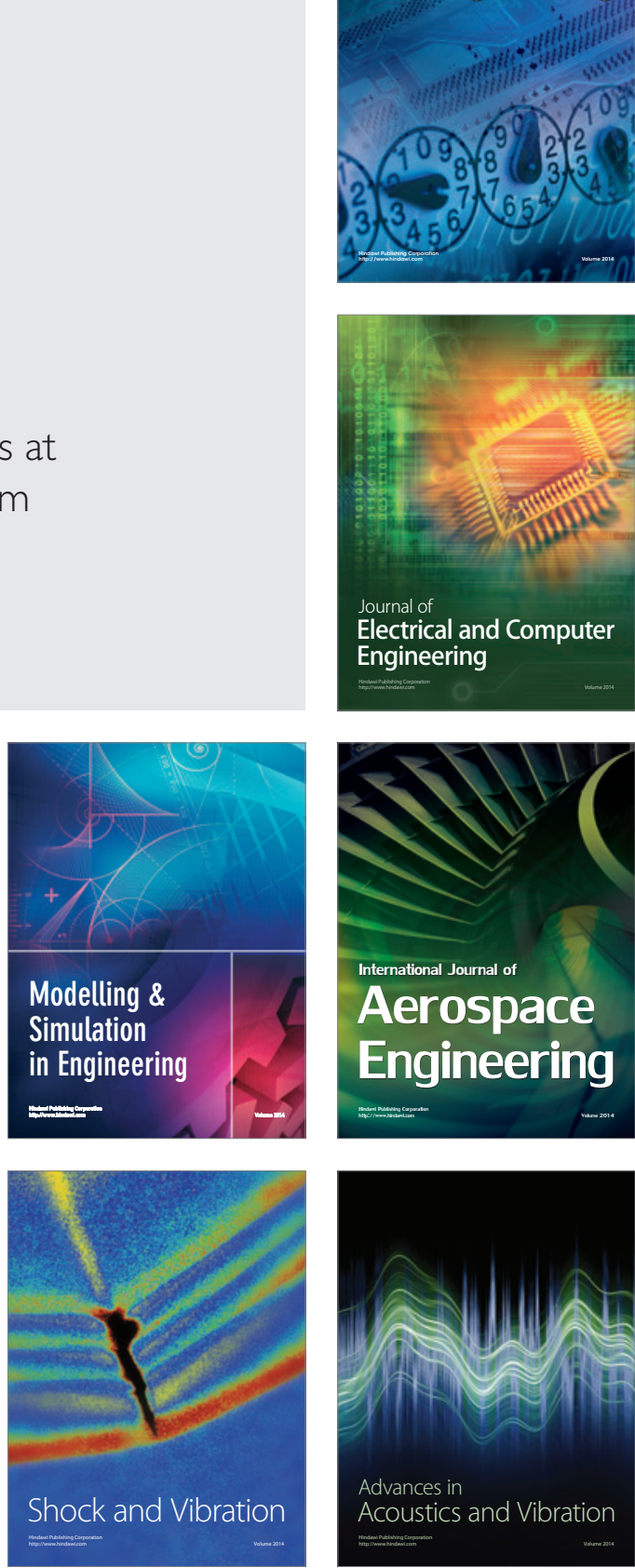